\title{
A Single-Step Synthesis of Azetidine-3-amines
}

\author{
Brian J. Wang ${ }^{\dagger}$ and Matthew A. J. Duncton*, \\ †Ryss Laboratories Inc., 29540 Kohoutek Way, Union City, CA 94587, United States \\ ‡Duncton, LLC, 851 Cherry Avenue, Suite 27/604, San Bruno, CA 94066, United States \\ KEYWORDS (Word Style "BG_Keywords"). If you are submitting your paper to a journal that requires keywords, provide \\ significant keywords to aid the reader in literature retrieval.
}

\begin{abstract}
The azetidine group is frequently encountered within contemporary medicinal chemistry where it is viewed as a privileged structure. However, the introduction of an azetidine can be synthetically challenging. Herein, a straight-forward one step synthesis of azetidine-3-amines, starting from a bench stable, commercial material is presented. The reaction tolerates functional groups commonly encountered in biological-, medicinal- and agro-chemistry, and proceeds in moderateto-high yield with secondary amines, and moderate-to-low yield with primary amines. The methodology compares favorably to recent alternative procedures and can be utilized in "any-stage" functionalization, including late-stage azetidinylation of approved drugs and other compounds with pharmacological activity.
\end{abstract}

The azetidine motif is one of the most important substructures in pharmaceuticals and is present in a number of approved drugs such as antibiotics, ${ }^{1}$ kinases $^{2}$ (e.g. baricitinib, cobimetinib, itacitinib), and other compound clas$\operatorname{ses}^{3}$ (e.g. thrombin inhibitor, ximelagatran/melagatran; Cachannel blocker, azelnidipine). The azetidine group is also attractive since it can restrict the conformation of an acyclic counterpart (rigidification), ${ }^{4}$ and lead to compounds with an improved pharmacokinetic or toxicity profile. ${ }^{5}$ Azetidine-3-amines (3-aminoazetidines, or azaazetidines) are a less well developed subset of azetidines, that nonetheless have found important applications in medicinal chemistry, being a substructure of JNJ-41443532 (a CCR2 antagonist), ${ }^{6}$ macrolide antibiotics, ${ }^{7}$ triple reuptake inhibitors $^{8}$ and kinase inhibitors, ${ }^{9}$ amongst others (Figure 1). ${ }^{10}$ Recently, there has been a renewed interest in the synthesis of azetidine-3-amines, by modifying ring-opening conditions of azabicyclobutane (ABB) with amine nucleophiles in a strain-release reaction. ${ }^{11,12}$ Other approaches to azetidine-3-amines are based upon reductive amination. ${ }^{8,13}$ Yet another synthesis of azetidine-3-amines, which has been used sporadically, is the direct displacement of an azetidine electrophile with an amine nucleophile. ${ }^{14}$ This approach is more frequently encountered in the patent literature, ${ }^{15}$ with a direct displacement of 1-benzhydrylazetidin3-yl methanesulfonate $\mathbf{1}$ being the most frequently encountered azetidine electrophile. ${ }^{14,15}$ The resulting 1benzhydrylazetidinazetidine-3-amine products can be easily deprotected to the parent azetidine. ${ }^{16,17}$ In other cases, it is also possible to transform the 1-benzyhydryl protecting group directly to a carbamoyl chloride. ${ }^{18}$ One of us has prior experience of the amine displacement reaction with compound $\mathbf{1 , 1 9}$ and also has an interest in the azetidine group within drug discovery. ${ }^{20}$ In this paper we undertake a detailed study of the reaction of compound 1 with amines to afford azetidine-3-amine products. We found a simple "mix-and-heat" approach could be used at any stage of a synthesis, including the late-stage functionalization of approved drugs and other substances with pharmacological activity. In addition, this simple displacement approach seems to compare favorably to related strain-release methodology, particularly in-terms of experimental setup, yield and scope of substrate that can be employed.
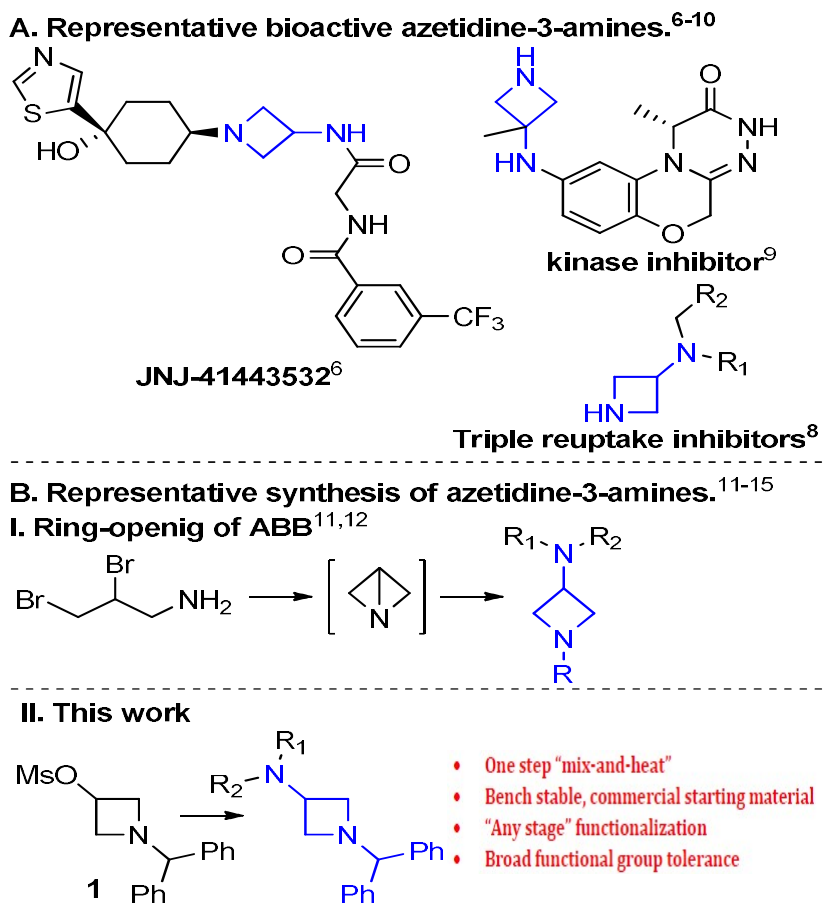

Figure 1. (A) Representative bioactive azetidine-3-amines. (B) Representative synthesis of azetidine-3-amines. 
We initiated our studies by examining the displacement of 1-benzhydrylazetidin-3-yl methanesulfonate $\mathbf{1}$ with simple amines. Compound $\mathbf{1}$ is a well-known, 21 commercially available intermediate, although for our studies, we prepared the material in a single batch starting from 1benzyhydryl-3-azetidinol. In our hands, 1 was stable at ambient (room) temperature for at least nine (9) months. Compound 1 was reacted with 1 equiv. piperidine and 1 equiv. of Hunig's base ( ${ }^{\mathrm{P}} \mathrm{PNEt}$ ) in $\mathrm{MeCN}$ at $80{ }^{\circ} \mathrm{C}$ to give a $33 \%$ yield of the desired displacement product 2 . A large improvement to the yield could be realized by using 2 equiv. of piperidine and omitting Hunig's base altogether. This afforded the desired product 2 in $72 \%$ isolated yield after purification by column chromatography (Scheme 1). The yield compares favorably to that reported when using strain-release methodology (56\% yield, reported in brackets in Scheme 1). ${ }^{11}$ Except for examples giving compounds 3 and 4, we employed 2 equiv. of amine for all subsequent reactions. As can be seen in Scheme 1, simple cyclic amines afforded fine isolated yields of products 2 to 8 . A more complex spirocyclic amine gave a lower $21 \%$ yield of product 9. Significantly, we could also utilize anilines in the displacement reaction, something that was not reported for the recently-reported strain-release technique. ${ }^{11,22}$ Thus, compound $\mathbf{1 0}$ was produced in 34\% isolated yield when 1,2,3,4-tetrahydroquinoline was used as a nucleophile. We also used acyclic secondary amines in the displacement. Again, good yields were obtained. For example, $N$-methylbenzylamine gave 11 in 69\% isolated yield ( $c f$. $46 \%$ using strain-release). Even the use of diisopropylamine, a very hindered secondary amine most commonly encountered in non-nucleophilic bases (e.g. LDA), but also a substructure of the herbicides diallate and triallate, afforded a $49 \%$ isolated yield of product 12. Late-stage azetidinylation of pharmacologically-active drug substances could also be undertaken in high-to-moderate yield. Thus, the 5-HT $2 \mathrm{C}$ agonist, 1-(3-trifluoromethylphenyl) piperazine (TFMPP), and the selective serotonin reuptake inhibitors, fluoxetine and sertraline, gave products 13 (87\%), 14 (79\%) and 15 (59\% cf. 45\% using strain-release).

Next, we sought to extend the methodology to the use of primary amines, since these could not be utilized in analogous strain-release methodology. ${ }^{23}$ The use of simple chain amines such as benzylamine, alpha-methylbenzylamine, or octylamine gave good yields of products 16 (48\%), 17 $(60 \%)$ and 18 (47\%). We then extended the methodology to the use of functionalized amines. Significantly, the reaction tolerated common functionality found in medicinal chemistry, such as difluoromethyl (19; 42\%), trifluoromethyl $(20 ; 44 \%)$, methoxy $(21 ; 33 \%)$ and even a free hydroxyl group $(22 ; 27 \%)$. The use of primary amines appended to a cyclic system were also successful giving products 23 (54\%), 24 (28\%) and 25 (27\%) in moderateto-low yield. In the case of products 23 and 24 a Bocprotected amine was unaffected in the reaction. Finally, we undertook a late-stage azetidinylation of 5methoxytryptamine (5-MT; aka, mexamine), which contains an unprotected indole nitrogen, to give 26 in $17 \%$ isolated yield.

The overall versatility and success of this straight-forward displacement reaction deserves some comment, especially in relation to complementary methodology such as the recently-reported strain-release approach to azetidinylation. ${ }^{11}$ The direct displacement described in this paper uses mild and very simple reaction conditions (mixing of two substances in reagent grade $\mathrm{MeCN}$ and heating to 80 ${ }^{\circ} \mathrm{C}$ ), with equipment that does not have to be flame-dried prior to use. In comparison, azetidinylation using strainrelease methodology requires the use of $\mathrm{PhLi}$, added dropwise and slowly, ${ }^{23}$ at $-78{ }^{\circ} \mathrm{C}$ to form azabicyclobutane (ABB), in preparation for a "spring-loaded" ring-opening. In a separate flame-dried flask, an amine nucleophile also needs to be activated with a turbo-Grignard (iPrMgCl.LiCl), with evolution of gas. ${ }^{23}$ After $2 \mathrm{~h}$, the "turbo-amide" is added dropwise to the pre-formed solution of ABB at $-78{ }^{\circ} \mathrm{C}$. It should be noted that the reaction is sensitive to the time used to form $\mathrm{ABB}$, with $2 \mathrm{~h}$ reaction time being optimal for maximal yield, ${ }^{23}$ so timings have to be quite wellcontrolled. The strain-release reaction seems to occur uneventfully overnight from $-78{ }^{\circ} \mathrm{C}$ to room temperature. However, quenching to give a Boc-azetidine product occurs at $0{ }^{\circ} \mathrm{C}$ and requires the slow addition of $\mathrm{Boc}_{2} \mathrm{O}$ in dry THF. $^{23}$ Unfortunately, carbamates and free alcohols are incompatible with the strain-release technique, ${ }^{23}$ whereas free alcohols, carbamates and an unprotected heterocyclic nitrogen (e.g. indole of 5-MT) are all tolerated in the direct displacement reaction described in this paper. We did not evaluate the use of amines containing a ketone group, sulfide, or amide functionality, which are also incompatible with strain-release methodology. ${ }^{23}$ Future work can ascertain whether such functionality is unaffected with the direct displacement reaction. The isolated yields obtained with a direct displacement appear to be slightly higher than an analogous reaction using strain-release methodology (e.g. isolated yields for 2, 3, 4, 7, 11 and 15; the yield for compound $\mathbf{6}$ was slightly lower than the strain-release approach). The higher yields may be reflective of the multioperational nature of experiments using strain-release methodology, as compared to a simple "mix-and-heat" approach for displacement methodology. Perhaps, the biggest difference between the displacement and strainrelease techniques, concerns the participation of primary amines. The use of primary amines in the displacement reaction was successful, giving moderate-to-low yields of product, whereas primary amines are incompatible with the strain-release method. ${ }^{23}$ Considering the above, we conclude that the direct displacement may offer advantages over strain-release methodology, especially interms of operational simplicity, scope of substrate and isolated yield. The facile nature of the displacement reaction, even for late-stage functionalization of unprotected starting materials, may appeal to those working in an industrial environment, where complexity (time) considerations are important.

In summary, this paper describes a simple one-step synthesis of azetidine-3-amines from a bench-stable commercial starting material. Both secondary and primary amines can successfully participate in the reaction, and the procedure tolerates common functionality such as ether, halide, difluoromethyl, trifluoromethyl, carbamate, unprotected heterocyle and even free hydroxyl groups. The methodology can be used for the late-stage functionalization of substances with pharmacological activity and compares favor- 
ably to complementary techniques such as strain-release azetidinylation. It is anticipated that the methodology described in this paper will find application in medicinal, biochemical, materials, polymer and agrochemical disciplines, where the azetidine group has shown to be a very useful and desirable motif.

Scheme 1. Scope of azetidinylation upon reaction of 1-benzhydrylazetidin-3-yl methanesulfonate 1 with amines.

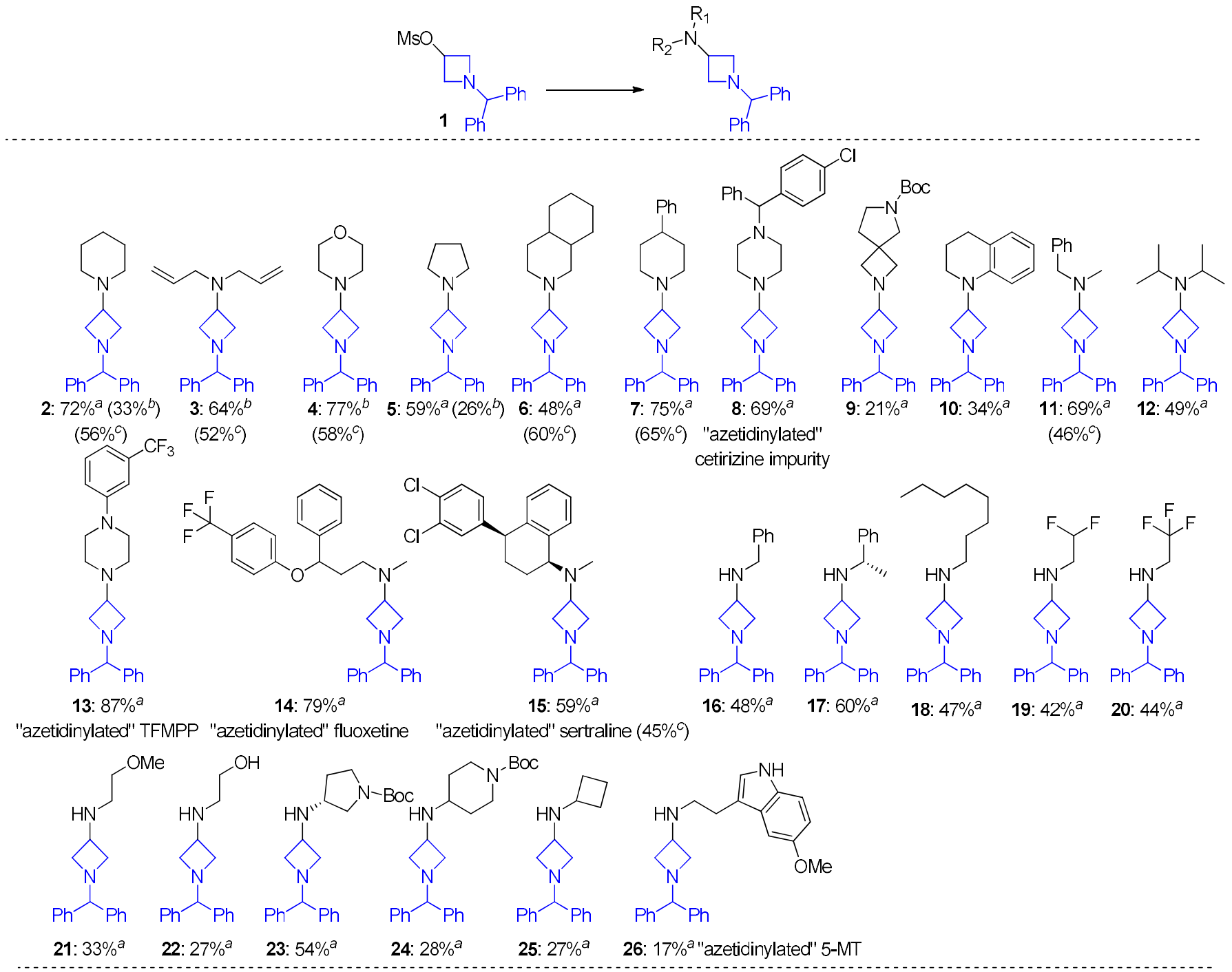

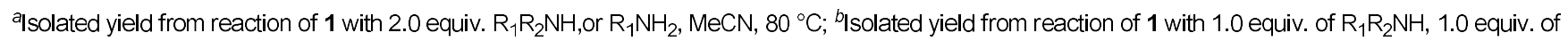
${ }^{i} \mathrm{Pr}_{2} \mathrm{NEt}, \mathrm{MeCN}, 80^{\circ} \mathrm{C}$; ' Yield of a analogous reaction using "strain-release" methodology to give an $\mathrm{N}$-Boc azetidine

\section{EXPERIMENTAL SECTION}

General. All reagents were purchased from commercial sources and used without further purification. All solvents were reagent, or HPLC grade. Analytical TLC was performed on silica gel 60 F254 plates and visualized by UV if possible, or by staining with $\mathrm{KMnO}_{4}$ dip, or phosphomolybdic acid in EtOH dip. Flash chromatography was carried out using an automated system with pre-packed silica columns. Yields refer to isolated yields of pure compounds. ${ }^{1} \mathrm{H}$ NMR and ${ }^{13} \mathrm{C}$ NMR spectra were recorded on a $300 \mathrm{MHz}$ spectrometer at ambient temperature. Chemical shifts are reported in parts per million ( $\mathrm{ppm}$ ) relative to deuterated solvent, or a TMS internal standard. Multiplicities are reported as follows: $\mathrm{s}=$ singlet; $\mathrm{d}=$ doublet, $\mathrm{t}=$ triplet; $\mathrm{dd}=$ doublet of doublets; $\mathrm{dt}=$ doublet of triplets; $\mathrm{m}=$ multiplet; $\mathrm{br}=$ broad; $\mathrm{f}=$ fine. High-resolution mass spectrometry was obtained on a Waters Xevo G2-XS QToF.

1-benzhydrylazetidin-3-yl methanesulfonate (1). ${ }^{21,24} \mathrm{MsCl}$ (3.9 $\mathrm{mL}, 50 \mathrm{mmol}$ ) was added dropwise to a solution of 1benzyhydryl-3-azetidinol (10 g, $42 \mathrm{mmol})$ and $\mathrm{Et}_{3} \mathrm{~N}(8.2$ $\mathrm{mL}, 59 \mathrm{mmol})$ in DCM $(125 \mathrm{~mL})$ at $0{ }^{\circ} \mathrm{C}$ (ice-water bath), and the mixture was stirred at $0{ }^{\circ} \mathrm{C}$ (ice-water bath), for 1 h. Saturated aqueous $\mathrm{NaHCO}_{3}$ was added. The phases were separated, and the aqueous layer was extracted with DCM (3 $\times 125 \mathrm{~mL}$ ). The combined organic layers were dried 
over $\mathrm{Na}_{2} \mathrm{SO}_{4}$, filtered, and concentrated to give the product as a solid (14.2 g). LC-MS: $317.88[\mathrm{M}+\mathrm{H}]^{+}$; ${ }^{1} \mathrm{H}-\mathrm{NMR}(300$ $\mathrm{MHz}_{\mathrm{CDCl}}$ ): $\delta$ 7.41-7.38 (m, 4H), 7.30-7.25 (m, 4H), 7.22$7.17(\mathrm{~m}, 2 \mathrm{H}), 5.10(\mathrm{~m}, 1 \mathrm{H}), 4.41(\mathrm{~s}, 1 \mathrm{H}), 3.67-3.62(\mathrm{~m}, 2 \mathrm{H})$, 3.23-3.18 (m, 2H), $2.98(\mathrm{~s}, 3 \mathrm{H})$.

General procedure for the synthesis of azetidine-3amines using 1 equiv. of amine. A solution of $1(630 \mathrm{mg}$, $2 \mathrm{mmol}$ ) in MeCN (9.5 mL) was treated with Et 2 NEt $(0.35$ $\mathrm{mL}, 2 \mathrm{mmol})$ and amine $(2 \mathrm{mmol})$. The reaction mixture was sealed and stirred at $80^{\circ} \mathrm{C}$ overnight. The mixture was concentrated, and the residue was dissolved in 1:1 EtOAc / hexanes $(30 \mathrm{~mL})$. The organic layer was washed with 1:1 $\mathrm{H}_{2} \mathrm{O}$ / brine $(30 \mathrm{~mL})$, brine $(30 \mathrm{~mL})$, dried over $\mathrm{MgSO}_{4}$, filtered, concentrated and purified by silica gel column chromatography to give the product.

$\mathrm{N}, \mathrm{N}$-diallyl-1-benzhydrylazetidin-3-amine (3). Purified using 0:1 to 3:7 EtOAc / hexanes as eluent to give a solid (408 mg, 64\% yield). LC-MS: $m / z=319.34[\mathrm{M}+\mathrm{H}]^{+} ;{ }^{1} \mathrm{H}-$ NMR (300 MHz, $\left.\mathrm{CDCl}_{3}\right): \delta$ 7.42-7.38 (m, 4H), 7.29-7.24 (m, $4 \mathrm{H}), 7.20-7.15(\mathrm{~m}, 2 \mathrm{H}), 5.88-5.74(\mathrm{~m}, 2 \mathrm{H}), 5.15-5.08(\mathrm{~m}$, $4 \mathrm{H}), 4.38(\mathrm{~s}, 1 \mathrm{H}), 3.43-3.27(\mathrm{~m}, 3 \mathrm{H}), 3.01(\mathrm{~d}, J=6.4 \mathrm{~Hz}, 4 \mathrm{H})$, 2.88-2.83 (m, 2H); ${ }^{13} \mathrm{C}-\mathrm{NMR}\left(75 \mathrm{MHz}^{\mathrm{CDCl}} 3\right): \delta 142.3$, 134.7, 128.5, 127.6, 127.2, 118.2, 78.5, 59.8, 54.3, 52.9; HRMS (ESI): $[\mathrm{M}+\mathrm{H}]^{+}$calc'd for $\mathrm{C}_{22} \mathrm{H}_{26} \mathrm{~N}_{2} \mathrm{~m} / \mathrm{z}$ 319.2174, found 319.2171.

4-(1-Benzhydrylazetidin-3-yl)morpholine (4). Purified using $0: 1$ to $1: 1$ EtOAc / hexanes as eluent to give an oil $(239 \mathrm{mg}$, $77 \%$ yield). LC-MS: $m / z=309.29[\mathrm{M}+\mathrm{H}]^{+} ;{ }^{1} \mathrm{H}-\mathrm{NMR}(300$ $\left.\mathrm{MHz}_{\mathrm{CDCl}}\right): \delta$ 7.42-7.40 (m, 4H), 7.28-7.24 (m, 4H), 7.20$7.15(\mathrm{~m}, 2 \mathrm{H}), 4.51(\mathrm{~s}, 1 \mathrm{H}), 3.42-3.39(\mathrm{~m}, 2 \mathrm{H}), 3.17(\mathrm{~m}, 3 \mathrm{H})$, $2.63(\mathrm{~m}, 4 \mathrm{H}), 1.88(\mathrm{~m}, 4 \mathrm{H}) ;{ }^{13} \mathrm{C}-\mathrm{NMR}(75 \mathrm{MHz}, \mathrm{CDCl} 3): \delta$ 142.2, 128.5, 127.6, 127.2, 78.3, 66.7, 58.0, 55.0, 50.3; HRMS (ESI): $[\mathrm{M}+\mathrm{H}]^{+}$calc'd for $\mathrm{C}_{20} \mathrm{H}_{24} \mathrm{~N}_{2} \mathrm{O} \mathrm{m} / z$ 309.1967, found 309.1968.

General procedure for the synthesis of azetidine-3amines using 2 equiv. of amine. A solution of 1 (317 mg, $1 \mathrm{mmol}$ ) in MeCN (5 mL) was treated with amine (2 $\mathrm{mmol})$. The reaction mixture was sealed and stirred at 80 ${ }^{\circ} \mathrm{C}$ overnight. The mixture was concentrated, and the residue was dissolved in 1:1 EtOAc / hexanes $(30 \mathrm{~mL})$. The organic layer was washed with $1: 1 \mathrm{H}_{2} \mathrm{O} /$ brine $(30 \mathrm{~mL})$, brine $(30 \mathrm{~mL})$, dried over $\mathrm{MgSO}_{4}$, filtered, concentrated and purified by silica gel column chromatography.

1-(1-Benzhydrylazetidin-3-yl)piperidine (2). Purified using $0: 1$ to $1: 4$ EtOAc / hexanes as eluent to give a solid (221 $\mathrm{mg}, 72 \%$ yield). LC-MS: $m / z=307.36[\mathrm{M}+\mathrm{H}]^{+} ;{ }^{1} \mathrm{H}-\mathrm{NMR}$ (300 MHz, $\left.\mathrm{CDCl}_{3}\right): \delta$ 7.41-7.38 (m, 4H), 7.27-7.22 (m, 4H), 7.17-7.13 (m, 2H), $4.42(\mathrm{~s}, 1 \mathrm{H}), 3.39(\mathrm{~m}, 2 \mathrm{H}), 2.88(\mathrm{~m}, 3 \mathrm{H})$, $2.17(\mathrm{~m}, 4 \mathrm{H}), 1.55-1.52(\mathrm{~m}, 4 \mathrm{H}), 1.41-1.40(\mathrm{~m}, 2 \mathrm{H}) .{ }^{13} \mathrm{C}-$ NMR (75 MHz, $\left.\mathrm{CDCl}_{3}\right): \delta 142.3,128.4,127.6,127.1,78.1$, 58.7, 55.4, 51.1, 25.4, 24.2; HRMS (ESI): [M+H] ${ }^{+}$calc'd for $\mathrm{C}_{21} \mathrm{H}_{26} \mathrm{~N}_{2} \mathrm{~m} / z$ 307.2174, found 307.2177.

1-(1-Benzhydrylazetidin-3-yl)pyrrolidine (5). Purified using 0:1 to 5:95 MeOH / DCM as eluent to give an oil $(174 \mathrm{mg}$, 59\% yield). LC-MS: $m / z=293.27[\mathrm{M}+\mathrm{H}]^{+} ;{ }^{1} \mathrm{H}-\mathrm{NMR}(300$ $\mathrm{MHz}_{\mathrm{CDCl}}$ ): $\delta$ 7.43-7.41 (m, 4H), 7.34-7.24 (m, 4H), 7.20$7.15(\mathrm{~m}, 2 \mathrm{H}), 4.43(\mathrm{~s}, 1 \mathrm{H}), 3.42-3.37(\mathrm{~m}, 2 \mathrm{H}), 3.11-2.95(\mathrm{~m}$, $3 \mathrm{H}), 2.40-2.38(\mathrm{~m}, 4 \mathrm{H}), 1.83-1.73(\mathrm{~m}, 4 \mathrm{H}) ;{ }^{13} \mathrm{C}-\mathrm{NMR}(75$ $\mathrm{MHz}_{\mathrm{CDCl}}$ ): $\delta 142.3,128.5,127.6,127.1,78.3,59.0,53.9$,
51.5, 23.5; HRMS (ESI): $[\mathrm{M}+\mathrm{H}]^{+}$calc'd for $\mathrm{C}_{20} \mathrm{H}_{24} \mathrm{~N}_{2} \mathrm{~m} / z$ 293.2018, found 293.2023.

2-(1-benzhydrylazetidin-3-yl)decahydroisoquinoline (6). After stirring at $80{ }^{\circ} \mathrm{C}$ overnight, the mixture was placed in a $-20{ }^{\circ} \mathrm{C}$ freezer for $16 \mathrm{~h}$, giving crystals. These were collected by filtration and rinsed with cold MeCN to give a crude solid, which was recrystallized from MeCN (x 2) to give a solid (173 mg, 48\% yield). LC-MS: $m / z=361.14$ $[\mathrm{M}+\mathrm{H}]^{+} ;{ }^{1} \mathrm{H}-\mathrm{NMR}\left(300 \mathrm{MHz}, \mathrm{CDCl}_{3}\right): \delta$ 7.42-7.40 (m, 4H), 7.29-7.24 (m, 4H), 7.21-7.15 (m, 2H), $4.43(\mathrm{~s}, 1 \mathrm{H}), 3.44-$ $3.38(\mathrm{~m}, 2 \mathrm{H}), 2.88-2.84(\mathrm{~m}, 3 \mathrm{H}), 2.22-2.05(\mathrm{~m}, 4 \mathrm{H}), 1.67-$ $1.34(\mathrm{~m}, 10 \mathrm{H}) ;{ }^{13} \mathrm{C}-\mathrm{NMR}\left(75 \mathrm{MHz}, \mathrm{CDCl}_{3}\right): \delta 142.4,128.5$, 127.6, 127.1, 78.3, 59.0, 58.8, 55.3, 33.9; HRMS (ESI): $[\mathrm{M}+\mathrm{H}]^{+}$calc'd for $\mathrm{C}_{25} \mathrm{H}_{32} \mathrm{~N}_{2} \mathrm{~m} / z$ 361.2644, found 361.2643.

1-(1-benzhydrylazetidin-3-yl)-4-phenylpiperidine (7). After stirring at $80{ }^{\circ} \mathrm{C}$ overnight, the mixture was placed in a -20 ${ }^{\circ} \mathrm{C}$ freezer for $16 \mathrm{~h}$, giving crystals. The solid was purified by silica gel column chromatography using $0: 1$ to $1: 4$ EtOAc / hexanes to give a solid (288 mg, 75\% yield). LCMS: $m / z=383.14[\mathrm{M}+\mathrm{H}]^{+} ;{ }^{1} \mathrm{H}-\mathrm{NMR}\left(300 \mathrm{MHz}, \mathrm{CDCl}_{3}\right): \delta$ 7.46-7.43 (m, 4H), 7.34-7.17 (m, 11H), $4.48(\mathrm{~s}, 1 \mathrm{H}), 3.49$ $3.45(\mathrm{~m}, 2 \mathrm{H}), 3.05-2.85(\mathrm{~m}, 5 \mathrm{H}), 2.55-2.44(\mathrm{~m}, 1 \mathrm{H}), 1.93-$ $1.71(\mathrm{~m}, 6 \mathrm{H}) ;{ }^{13} \mathrm{C}-\mathrm{NMR}\left(75 \mathrm{MHz}, \mathrm{CDCl}_{3}\right): \delta 146.3,142.3$, 128.5, 127.6, 127.2, 126.9, 126.3, 78.1, 58.8, 55.3, 51.1, 42.6, 32.9; HRMS (ESI): $[\mathrm{M}+\mathrm{H}]^{+}$calc'd for $\mathrm{C}_{27} \mathrm{H}_{30} \mathrm{~N}_{2} \mathrm{~m} / \mathrm{z}$ 383.2487, found 383.2475.

\section{1-(1-Benzhydrylazetidin-3-yl)-4-((4-}

chlorophenyl)(phenyl)methyl)piperazine (8). Purified using 5:95 to 45:55 EtOAc / hexanes as eluent to give a solid (355 mg, 69\% yield). LC-MS: $m / z=508.07[\mathrm{M}+\mathrm{H}]^{+} ;{ }^{1} \mathrm{H}-$ NMR (300 MHz, $\left.\mathrm{CDCl}_{3}\right): \delta$ 7.40-7.31 (m, 8H), 7.28-7.15 (m, $11 \mathrm{H}), 4.40(\mathrm{~s}, 1 \mathrm{H}), 4.21(\mathrm{~s}, 1 \mathrm{H}), 3.41-3.37(\mathrm{~m}, 2 \mathrm{H}), 3.01-$ $2.95(\mathrm{~m}, 1 \mathrm{H}), 2.89-2.85(\mathrm{~m}, 2 \mathrm{H}), 2.32$ (m, 8H; 13C-NMR (75 $\left.\mathrm{MHz}, \mathrm{CDCl}_{3}\right): \delta 142.2,142.1,141.3,132.6,129.3,128.7$, $128.69,128.5,127.9,127.6,127.3,127.2,78.2,75.4,58.3$, 55.0, 51.3, 50.2; HRMS (ESI): [M+H] ${ }^{+}$calc'd for $\mathrm{C}_{33} \mathrm{H}_{34} \mathrm{ClN}_{3}$ $m / z$ 508.2519, found 508.2522.

Tert-butyl 2-(1-benzhydrylazetidin-3-yl)-2,6diazaspiro[3.4]octane-6-carboxylate (9). Purified using 1:1 to 1:0 EtOAc / hexanes as eluent to give an oil (94 mg, $21 \%$ yield). LC-MS: $m / z=434.10[\mathrm{M}+\mathrm{H}]^{+} ;{ }^{1} \mathrm{H}-\mathrm{NMR}(300 \mathrm{MHz}$, $\left.\mathrm{CDCl}_{3}\right): \delta$ 7.41-7.38 (m, 4H), 7.28-7.24 (m, 4H), 7.19-7.15 $(\mathrm{m}, 2 \mathrm{H}), 4.35$ (s, 1H), 3.41 (s, 2H), 3.37-3.22 (m, 5H), 3.16$3.12(\mathrm{~m}, 4 \mathrm{H}), 2.93-2.89(\mathrm{~m}, 2 \mathrm{H}), 2.06-1.95(\mathrm{~m}, 2 \mathrm{H}), 1.45(\mathrm{~s}$, 9H); ${ }^{13} \mathrm{C}-\mathrm{NMR}\left(75 \mathrm{MHz}, \mathrm{CDCl}_{3}\right): \delta 154.6,142.3,128.5$, $127.5,127.2,79.4,78.2,60.4,60.2$, 56.7, 55.9, 55.2, 54.6, 44.9, 44.4, 40.6, 39.7, 36.3, 35.4, 28.6; HRMS (ESI): [M+H] ${ }^{+}$ calc'd for $\mathrm{C}_{27} \mathrm{H}_{35} \mathrm{~N}_{3} \mathrm{O}_{2} \mathrm{~m} / z$ 434.2808, found 434.2793.

1-(1-benzhydrylazetidin-3-yl)-1,2,3,4-tetrahydroquinoline (10). After stirring at $80{ }^{\circ} \mathrm{C}$ overnight, the mixture was placed in a $0-5{ }^{\circ} \mathrm{C}$ fridge for $16 \mathrm{~h}$, giving crystals. The solid was purified by silica gel column chromatography using $0: 1$ to $1: 9 \mathrm{EtOAc}+1 \% \mathrm{Et}_{3} \mathrm{~N} /$ hexanes $+1 \% \mathrm{Et}_{3} \mathrm{~N}$ as eluent to give a solid (121 mg, 34\% yield). LC-MS: $m / z=355.05$ $[\mathrm{M}+\mathrm{H}]^{+} ;{ }^{1} \mathrm{H}-\mathrm{NMR}\left(300 \mathrm{MHz}, \mathrm{CDCl}_{3}\right): \delta$ 7.45-7.42 (m, 4H), 7.31-7.26 (m, 4H), 7.22-7.16 (m, 2H), 7.00-6.95 (m, 2H), $6.64(\mathrm{t}, J=7.3 \mathrm{~Hz}, 1 \mathrm{H}), 6.34(\mathrm{~d}, J=8.2 \mathrm{~Hz}, 1 \mathrm{H}), 4.36(\mathrm{~s}, 1 \mathrm{H})$, $4.05(\mathrm{~m}, 1 \mathrm{H}), 3.67(\mathrm{t}, J=7.3 \mathrm{~Hz}, 2 \mathrm{H}), 3.03(\mathrm{~m}, 4 \mathrm{H}), 2.74(\mathrm{t}, J$ $=6.4 \mathrm{~Hz}, 2 \mathrm{H}), 1.94(\mathrm{~m}, 2 \mathrm{H}) ;{ }^{13} \mathrm{C}-\mathrm{NMR}\left(75 \mathrm{MHz} \mathrm{CDCl}_{3}\right): \delta$ $145.9,142.1,128.9,128.6,127.6,127.3,126.9,124.7$, 
117.4, 112.0, 78.6, 59.7, 49.7, 44.5, 27.7, 22.9; HRMS (ESI): $[\mathrm{M}+\mathrm{H}]^{+}$calc'd for $\mathrm{C}_{25} \mathrm{H}_{26} \mathrm{~N}_{2} \mathrm{~m} / \mathrm{z} 355.2174$, found 355.2176 .

1-Benzhydryl-N-benzyl-N-methylazetidin-3-amine

(11).

After stirring at $80^{\circ} \mathrm{C}$ overnight, the mixture was placed in a $-20{ }^{\circ} \mathrm{C}$ freezer for $16 \mathrm{~h}$, giving crystals. These were collected by filtration and carefully rinsed with a small amount of cold MeCN to give a solid (239 mg, 69\% yield). LC-MS: $m / z=343.02[\mathrm{M}+\mathrm{H}]^{+} ;{ }^{1} \mathrm{H}-\mathrm{NMR}\left(300 \mathrm{MHz}, \mathrm{CDCl}_{3}\right): \delta$ 7.44-7.41 (m, 4H), 7.33-7.16 (m, 11H), $4.42(\mathrm{~s}, 1 \mathrm{H}), 3.45$ (t, $J=6.7 \mathrm{~Hz}, 2 \mathrm{H}), 3.33(\mathrm{~s}, 2 \mathrm{H}), 3.11(\mathrm{~m}, 1 \mathrm{H}), 2.91(\mathrm{t}, J=7.0 \mathrm{~Hz}$, $2 \mathrm{H}), 1.97(\mathrm{~s}, 3 \mathrm{H}) ;{ }^{13} \mathrm{C}-\mathrm{NMR}\left(75 \mathrm{MHz}, \mathrm{CDCl}_{3}\right): \delta 142.3,137.7$, 129.5, 128.5, 128.3, 127.6, 127.24, 127.21, 78.5, 59.2, 59.0, 54.7, 38.3; HRMS (ESI): $[\mathrm{M}+\mathrm{H}]^{+}$calc'd for $\mathrm{C}_{24} \mathrm{H}_{26} \mathrm{~N}_{2} \mathrm{~m} / \mathrm{z}$ 343.2174, found 343.2172.

1-Benzhydryl-N,N-diisopropylazetidin-3-amine (12). After stirring at $80^{\circ} \mathrm{C}$ overnight, the mixture was placed in a $0-5$ ${ }^{\circ} \mathrm{C}$ fridge for $16 \mathrm{~h}$, giving crystals, which were washed with cold MeCN to give a solid (158 mg, 49\% yield). LC-MS: $\mathrm{m} / \mathrm{z}$ $=323.09[\mathrm{M}+\mathrm{H}]^{+} ;{ }^{1} \mathrm{H}-\mathrm{NMR}\left(300 \mathrm{MHz}, \mathrm{CDCl}_{3}\right): \delta$ 7.43-7.40 (m, 4H), 7.29-7.24 (m, 4H), 7.21-7.16 (m, 2H), $4.37(\mathrm{~s}, 1 \mathrm{H})$, $3.71(\mathrm{~m}, 1 \mathrm{H}), 3.42-3.37(\mathrm{~m}, 2 \mathrm{H}), 2.94(\mathrm{~m}, 2 \mathrm{H}), 2.83(\mathrm{t}, J=$ $7.6 \mathrm{~Hz}, 2 \mathrm{H}), 0.96(\mathrm{~d}, J=6.5 \mathrm{~Hz}, 12 \mathrm{H}) ;{ }^{13} \mathrm{C}-\mathrm{NMR}(75 \mathrm{MHz}$, $\left.\mathrm{CDCl}_{3}\right): \delta 142.4,128.5,127.6,127.1,78.4,61.3,47.0,46.5$, 21.3; HRMS (ESI): [M+H] $]^{+}$calc'd for $\mathrm{C}_{22} \mathrm{H}_{30} \mathrm{~N}_{2} \mathrm{~m} / \mathrm{z}$ 323.2487, found 323.2488.

1-(1-Benzhydrylazetidin-3-yl)-4-(3-

(trifluoromethyl)phenyl)piperazine (13). After stirring at 80 ${ }^{\circ} \mathrm{C}$ overnight, the mixture was cooled and treated with EtOAc $(10 \mathrm{~mL})$, giving a solid, which was collected by filtration. The filtrate was concentrated, and the residue was purified by silica gel column chromatography using 5:95 to 3:7 EtOAc / hexanes as eluent to a solid $(397 \mathrm{mg}, 87 \%$ yield). LC-MS: $m / z=452.10[\mathrm{M}+\mathrm{H}]^{+} ;{ }^{1} \mathrm{H}-\mathrm{NMR}(300 \mathrm{MHz}$, $\left.\mathrm{CDCl}_{3}\right): \delta$ 7.44-7.41 (m, $\left.4 \mathrm{H}\right), 7.36-7.26(\mathrm{~m}, 5 \mathrm{H}), 7.22-7.17$ (m, 2H), 7.08-7.02 (m, 3H), $4.44(\mathrm{~s}, 1 \mathrm{H}), 3.45(\mathrm{~m}, 2 \mathrm{H}), 3.25-$ $3.22(\mathrm{t}, J=5.0 \mathrm{~Hz}, 4 \mathrm{H}), 3.04-2.95(\mathrm{~m}, 3 \mathrm{H}), 2.47-2.44(\mathrm{t}, J=$ $5.0 \mathrm{~Hz}, 4 \mathrm{H}) ;{ }^{13} \mathrm{C}-\mathrm{NMR}\left(75 \mathrm{MHz}, \mathrm{CDCl}_{3}\right): \delta 151.4,142.1$, $131.5(\mathrm{q}, J=32 \mathrm{~Hz}, 1 \mathrm{C}), 129.7,128.6,127.6,127.3,124.4$ $(\mathrm{q}, J=272 \mathrm{~Hz}, 1 \mathrm{C}), 118.8,116.0(\mathrm{q}, J=3 \mathrm{~Hz}, 1 \mathrm{C}), 112.3(\mathrm{q}, J$ $=4 \mathrm{~Hz}, 1 \mathrm{C}), 78.3,58.2,54.8,49.8,48.4 ;{ }^{19} \mathrm{~F}-\mathrm{NMR}(282 \mathrm{MHz}$, $\mathrm{CDCl}_{3}$ ): $\delta$-62.7; HRMS (ESI): $[\mathrm{M}+\mathrm{H}]^{+}$calc'd for $\mathrm{C}_{27} \mathrm{H}_{28} \mathrm{~F}_{3} \mathrm{~N}_{3}$ $\mathrm{m} / \mathrm{z}$ 452.2314, found 452.2302 .

1-Benzhydryl-N-methyl-N-(3-phenyl-3-(4-

(trifluoromethyl)phenoxy)propyl)azetidin-3-amine

(14).

Purified using 5:95 to 3:7 EtOAc / hexanes as eluent to give as an oil (423 mg, 79\% yield). LC-MS: $\mathrm{m} / z=531.10$ $[\mathrm{M}+\mathrm{H}]^{+} ;{ }^{1} \mathrm{H}-\mathrm{NMR}\left(300 \mathrm{MHz}, \mathrm{CDCl}_{3}\right): \delta$ 7.44-7.16 $(\mathrm{m}, 17 \mathrm{H})$, $6.88(\mathrm{~d}, J=8.8 \mathrm{~Hz}, 2 \mathrm{H}), 5.29-5.25(\mathrm{~m}, 1 \mathrm{H}), 4.22(\mathrm{~s}, 1 \mathrm{H})$, 3.38-3.36 (m, 2H), 3.03-2.99 (m, 1H), 2.79-2.71 (m, 2H), 2.47-2.38 (m, 1H), 2.32-2.24 (m, 1H), 2.15-2.03 (m, 4H), 1.99-1.90 (m, $1 \mathrm{H}) ;{ }^{13} \mathrm{C}-\mathrm{NMR}\left(75 \mathrm{MHz}, \mathrm{CDCl}_{3}\right): \delta 160.8$, $142.24,142.16,141.2,128.9,128.52,128.49,127.9,127.6$, $127.2,126.9$ (q, $J=4 \mathrm{~Hz}, 2 \mathrm{C}), 125.9,124.5(\mathrm{q}, J=271 \mathrm{~Hz}$, 1C), 122.8 (q, $J=33 \mathrm{~Hz}, 1 \mathrm{C}), 115.9,78.6,78.3,59.22,59.16$, 55.2, 50.7, 38.5, 36.3; ${ }^{19} \mathrm{~F}-\mathrm{NMR}\left(282 \mathrm{MHz}, \mathrm{CDCl}_{3}\right): \delta-61.5$; HRMS (ESI): $[\mathrm{M}+\mathrm{H}]^{+}$calc'd for $\mathrm{C}_{33} \mathrm{H}_{33} \mathrm{~F}_{3} \mathrm{~N}_{2} \mathrm{O} \mathrm{m} / z$ 531.2623, found 531.2625 .

1-Benzhydryl-N-((1S,4S)-4-(3,4-dichlorophenyl)-1,2,3,4tetrahydronaphthalen-1-yl)-N-methylazetidin-3-amine (15). Purified using 0:1 to 1:4 EtOAc / hexanes as eluent to give a solid (313 mg, 59\% yield). LC-MS: $m / z=527.17[\mathrm{M}+\mathrm{H}]^{+}$;
${ }^{1} \mathrm{H}-\mathrm{NMR}\left(300 \mathrm{MHz}, \mathrm{CDCl}_{3}\right): \delta 7.80(\mathrm{~d}, J=7.6 \mathrm{H}, 1 \mathrm{H}), 7.45-$ $7.42(\mathrm{~m}, 4 \mathrm{H}), 7.31-7.25(\mathrm{~m}, 6 \mathrm{H}), 7.22-7.11(\mathrm{~m}, 3 \mathrm{H}), 7.07$ (s, $1 \mathrm{H}), 6.87(\mathrm{~d}, J=7.6 \mathrm{~Hz}, 1 \mathrm{H}), 6.79(\mathrm{~d}, J=6.4 \mathrm{~Hz}, 1 \mathrm{H}), 4.38(\mathrm{~s}$, $1 \mathrm{H}), 4.12-4.08(\mathrm{~m}, 1 \mathrm{H}), 3.76(\mathrm{t}, J=7.9 \mathrm{~Hz}, 1 \mathrm{H}), 3.57-3.51$ (m, 1H), 3.43-3.41 (m, 2H), 2.89-2.88 (m, 2H), 2.12-1.90 $(\mathrm{m}, 5 \mathrm{H}), 1.59-1.52(\mathrm{~m}, 2 \mathrm{H}) ;{ }^{13} \mathrm{C}-\mathrm{NMR}\left(75 \mathrm{MHz}, \mathrm{CDCl}_{3}\right): \delta$ $147.5,142.3,139.0,138.2,132.2,130.8,130.3,130.0$, 130.0, 128.5, 128.3, 127.6, 127.2, 127.1, 126.9, 78.7, 59.6, 59.0, 58.3, 51.6, 43.6, 32.5, 30.2, 15.8; HRMS (ESI): $[\mathrm{M}+\mathrm{H}]^{+}$ calc'd for $\mathrm{C}_{33} \mathrm{H}_{32} \mathrm{Cl}_{2} \mathrm{~N}_{2} \mathrm{~m} / \mathrm{z}$ 527.2021, found 527.2021.

1-Benzhydryl-N-benzylazetidin-3-amine (16). Purified using 1:9 to 1:0 EtOAc $+1 \% \mathrm{Et}_{3} \mathrm{~N} /$ hexanes $+1 \% \mathrm{Et}_{3} \mathrm{~N}$ as eluent to give a solid (159 $\mathrm{mg}, 48 \%$ yield). LC-MS: $\mathrm{m} / z=329.21$ $[\mathrm{M}+\mathrm{H}]^{+} ;{ }^{1} \mathrm{H}-\mathrm{NMR}\left(300 \mathrm{MHz}, \mathrm{CDCl}_{3}\right): \delta$ 7.43-7.17 (m, 15H), $4.34(\mathrm{~s}, 1 \mathrm{H}), 3.71(\mathrm{~s}, 2 \mathrm{H}), 3.57-3.49(\mathrm{~m}, 3 \mathrm{H}), 2.78-2.75(\mathrm{~m}$, $2 \mathrm{H}) ;{ }^{13} \mathrm{C}$-NMR $\left(75 \mathrm{MHz}, \mathrm{CDCl}_{3}\right): \delta 142.3,140.0,128.6$, 128.5, 128.3, 127.5, 127.2, 78.6, 61.9, 51.7, 48.4; HRMS (ESI): $[\mathrm{M}+\mathrm{H}]+$ calc'd for $\mathrm{C}_{23} \mathrm{H}_{24} \mathrm{~N}_{2} \mathrm{~m} / \mathrm{z}$ 329.2018, found 329.2015 .

(S)-1-benzhydryl-N-(1-phenylethyl)azetidin-3-amine (17). Purified using 0:1 to 3:7 EtOAc / hexanes as eluent to give as an oil $(208 \mathrm{mg}, 60 \%$ yield). LC-MS: $\mathrm{m} / z=343.06$ $[\mathrm{M}+\mathrm{H}]^{+}$; ${ }^{1} \mathrm{H}-\mathrm{NMR}\left(300 \mathrm{MHz}, \mathrm{CDCl}_{3}\right): \delta$ 7.40-7.36 (m, 4H), 7.33-7.15 (m, 11H), $4.29(\mathrm{~s}, 1 \mathrm{H}), 3.73(\mathrm{q}, J=6.4 \mathrm{~Hz}, 1 \mathrm{H})$, $3.50-3.31(\mathrm{~m}, 3 \mathrm{H}), 2.72(\mathrm{t}, J=6.4 \mathrm{~Hz}, 1 \mathrm{H}), 2.62(\mathrm{t}, J=6.4 \mathrm{~Hz}$, $1 \mathrm{H}), 1.34(\mathrm{~d}, J=7.0 \mathrm{~Hz}, 3 \mathrm{H}) ;{ }^{13} \mathrm{C}-\mathrm{NMR}\left(75 \mathrm{MHz}, \mathrm{CDCl}_{3}\right): \delta$ $145.2,142.3,128.55,128.5,127.5,127.5,127.2,127.1$, 127.1, 126.7, 78.6, 62.3, 62.3, 56.5, 47.0, 24.0; HRMS (ESI): $[\mathrm{M}+\mathrm{H}]^{+}$calc'd for $\mathrm{C}_{24} \mathrm{H}_{26} \mathrm{~N}_{2} \mathrm{~m} / \mathrm{z} 343.2174$, found 343.2173.

1-Benzhydryl-N-octylazetidin-3-amine (18). After stirring at $80{ }^{\circ} \mathrm{C}$ overnight, the mixture was placed in a $0-5{ }^{\circ} \mathrm{C}$ fridge for $16 \mathrm{~h}$, giving crystals. The filtrate was purified by silica gel column chromatography using 1:4 to 3:7 EtOAc / hexanes as eluent to give a solid (165 $\mathrm{mg}, 47 \%$ yield). LC-MS: $m / z=351.14[\mathrm{M}+\mathrm{H}]^{+} ;{ }^{1} \mathrm{H}-\mathrm{NMR}\left(300 \mathrm{MHz}, \mathrm{CDCl}_{3}\right): \delta 7.42-$ $7.38(\mathrm{~m}, 4 \mathrm{H}), 7.29-7.24(\mathrm{~m}, 4 \mathrm{H}), 7.21-7.15(\mathrm{~m}, 2 \mathrm{H}), 4.32$ (s, $1 \mathrm{H}), 3.54-3.42(\mathrm{~m}, 3 \mathrm{H}), 2.74-2.70(\mathrm{~m}, 2 \mathrm{H}), 2.50(\mathrm{t}, J=7.3$ $\mathrm{Hz}, 2 \mathrm{H}), 1.45-1.41(\mathrm{~m}, 3 \mathrm{H}), 1.32-1.20(\mathrm{~m}, 10 \mathrm{H}), 0.88(\mathrm{t}, J=$ $6.7 \mathrm{~Hz}, 3 \mathrm{H}) ;{ }^{13} \mathrm{C}-\mathrm{NMR}\left(75 \mathrm{MHz}, \mathrm{CDCl}_{3}\right): \delta 142.3,128.5$, 127.6, 127.2, 78.7, 62.1, 48.9, 47.7, 31.9, 30.4, 29.6, 29.4, 27.5, 22.8, 14.2; HRMS (ESI): $[\mathrm{M}+\mathrm{H}]^{+}$calc'd for $\mathrm{C}_{24} \mathrm{H}_{34} \mathrm{~N}_{2}$ $\mathrm{m} / \mathrm{z} 351.2800$, found 351.2794 .

1-Benzhydryl-N-(2,2-difluoroethyl)azetidin-3-amine (19). After stirring at $80^{\circ} \mathrm{C}$ overnight, the mixture was placed in a $0-5{ }^{\circ} \mathrm{C}$ fridge for $16 \mathrm{~h}$, giving crystals. The filtrate was purified by silica gel column chromatography using 0:1 to 1:9 $\mathrm{MeOH} / \mathrm{DCM}$ as eluent to give an oil $(128 \mathrm{mg}, 42 \%$ yield). LC-MS: $m / z=302.99[\mathrm{M}+\mathrm{H}]^{+} ;{ }^{1} \mathrm{H}-\mathrm{NMR}(300 \mathrm{MHz}$, $\left.\mathrm{CDCl}_{3}\right): \delta$ 7.41-7.37 (m, 4H), 7.29-7.25 (m, 4H), 7.21-7.16 (m, 2H), 5.98-5.57 (m, 1H), 4.31 (s, 1H), 3.51-3.46 (m, 3H), 2.97-2.85 (m, 2H), 2.77-2.70 (m, 2H); ${ }^{13} \mathrm{C}-\mathrm{NMR}(75 \mathrm{MHz}$, $\left.\mathrm{CDCl}_{3}\right): \delta 142.1,128.6,127.5,127.2,115.7(\mathrm{t}, J=241 \mathrm{~Hz}$, 1C), 78.5, 61.7, $49.7(\mathrm{t}, J=24 \mathrm{~Hz}, 1 \mathrm{C}), 49.0 ;{ }^{19} \mathrm{~F}-\mathrm{NMR}(282$ $\mathrm{MHz}, \mathrm{CDCl}_{3}$ ): $\delta-122.1(\mathrm{dt}, J=56,15 \mathrm{~Hz}, 2 \mathrm{~F}$ ); HRMS (ESI): $[\mathrm{M}+\mathrm{H}]^{+}$calc'd for $\mathrm{C}_{18} \mathrm{H}_{20} \mathrm{~F}_{2} \mathrm{~N}_{2} \mathrm{~m} / z$ 303.1673, found 303.1673.

1-Benzhydryl-N-(2,2,2-trifluoroethyl)azetidin-3-amine (20). Purified using 0:1 to 5:95 MeOH / DCM as eluent to afford a crude solid, which was purified again using 0:1 to $3: 7$ $\mathrm{EtOAc}+1 \% \mathrm{Et}_{3} \mathrm{~N} /$ hexanes $+1 \% \mathrm{Et}_{3} \mathrm{~N}$ as eluent to give a solid (141 mg, 44\% yield); LC-MS: $m / z=320.95[\mathrm{M}+\mathrm{H}]^{+}$; 
1H-NMR (300 MHz, $\left.\mathrm{CDCl}_{3}\right): \delta$ 7.40-7.38 (m, 4H), 7.30-7.25 (m, 4H), 7.21-7.16 (m, 2H), $4.30(\mathrm{~s}, 1 \mathrm{H}), 3.52-3.51(\mathrm{~m}, 3 \mathrm{H})$, $3.14(\mathrm{q}, J=9.4 \mathrm{~Hz}, 2 \mathrm{H}), 2.74(\mathrm{~m}, 2 \mathrm{H}) ;{ }^{13} \mathrm{C}-\mathrm{NMR}(75 \mathrm{MHz}$, $\left.\mathrm{CDCl}_{3}\right): \delta 142.1,128.6,127.5,127.3,125.3(\mathrm{q}, J=279 \mathrm{~Hz}$, 1C), 78.5, 62.0, 49.1 (q, $J=31 \mathrm{~Hz}, 1 \mathrm{C}), 49.0 ;{ }^{19} \mathrm{~F}-\mathrm{NMR}(282$ $\left.\mathrm{MHz}, \mathrm{CDCl}_{3}\right): \delta-72.2(\mathrm{t}, J=9 \mathrm{~Hz}, 3 \mathrm{~F})$; HRMS (ESI): $[\mathrm{M}+\mathrm{H}]^{+}$ calc'd for $\mathrm{C}_{18} \mathrm{H}_{19} \mathrm{~F}_{3} \mathrm{~N}_{2} \mathrm{~m} / \mathrm{z} 321.1579$, found 321.1578.

1-Benzhydryl-N-(2-methoxyethyl)azetidin-3-amine (21). Purified using 0:1 to 5:95 MeOH / DCM $+1 \% \mathrm{Et}_{3} \mathrm{~N}$ as eluent to give an oil, which after cooling at $-20^{\circ} \mathrm{C}$ solidified to a solid (99 mg, 33\% yield). LC-MS: $m / z=297.04[\mathrm{M}+\mathrm{H}]^{+} ;{ }^{1} \mathrm{H}-$ NMR $\left(300 \mathrm{MHz}, \mathrm{CDCl}_{3}\right): \delta$ 7.41-7.37 (m, 4H), 7.29-7.23 (m, $4 \mathrm{H}), 7.20-7.15(\mathrm{~m}, 2 \mathrm{H}), 4.32(\mathrm{~s}, 1 \mathrm{H}), 3.54-3.48(\mathrm{~m}, 3 \mathrm{H}), 3.44$ $(\mathrm{t}, J=5.3 \mathrm{~Hz}, 2 \mathrm{H}), 3.34(\mathrm{~s}, 3 \mathrm{H}), 2.76-2.68(\mathrm{~m}, 4 \mathrm{H}) ;{ }^{13} \mathrm{C}-\mathrm{NMR}$ $\left(75 \mathrm{MHz}, \mathrm{CDCl}_{3}\right): \delta 142.3,128.5,127.5,127.1,78.6,72.1$, 61.9, 58.9, 48.8, 47.0; HRMS (ESI): $[\mathrm{M}+\mathrm{H}]^{+}$calc'd for $\mathrm{C}_{19} \mathrm{H}_{24} \mathrm{~N}_{2} \mathrm{O} / \mathrm{m} / z$ 297.1967, found 297.1972.

2-((1-Benzhydrylazetidin-3-yl)amino)ethanol (22). Purified using 1:9 to 1:0 EtOAc $+1 \% \mathrm{Et}_{3} \mathrm{~N} /$ hexanes $+1 \% \mathrm{Et}_{3} \mathrm{~N}$ as eluent to give a solid $(78 \mathrm{mg}, 27 \%$ yield); LC-MS: $\mathrm{m} / z=$ $282.96[\mathrm{M}+\mathrm{H}]^{+} ;{ }^{1} \mathrm{H}-\mathrm{NMR}\left(300 \mathrm{MHz}, \mathrm{CDCl}_{3}\right): \delta$ 7.41-7.38 (m, $4 \mathrm{H}), 7.30-7.24(\mathrm{~m}, 4 \mathrm{H}), 7.21-7.18(\mathrm{~m}, 2 \mathrm{H}), 4.33(\mathrm{~s}, 1 \mathrm{H}), 3.62$ $(\mathrm{t}, J=5.0 \mathrm{~Hz}, 2 \mathrm{H}), 3.50-3.49(\mathrm{~m}, 3 \mathrm{H}), 2.80-2.79(\mathrm{~m}, 2 \mathrm{H})$, $2.70(\mathrm{t}, J=5.0 \mathrm{~Hz}, 2 \mathrm{H}) ;{ }^{13} \mathrm{C}-\mathrm{NMR}\left(75 \mathrm{MHz}, \mathrm{CDCl}_{3}\right): \delta 142.0$, 128.5, 127.5, 127.2, 78.5, 61.3, 60.9, 48.8, 48.4; HRMS (ESI): $[\mathrm{M}+\mathrm{H}]^{+}$calc'd for $\mathrm{C}_{18} \mathrm{H}_{22} \mathrm{~N}_{2} \mathrm{O} \mathrm{m} / \mathrm{z}$ 283.1811, found 283.1811.

(R)-tert-butyl

3-((1-benzhydrylazetidin-3yl)amino)pyrrolidine-1-carboxylate (23). After stirring at $80{ }^{\circ} \mathrm{C}$ overnight, the mixture was placed in a $-20^{\circ} \mathrm{C}$ freezer for $16 \mathrm{~h}$, giving crystals. The filtrate was purified by silica gel column chromatography using 0:1 to 9:1 EtOAc / hexanes as eluent to give a solid (221 $\mathrm{mg}, 54 \%$ yield). LC-MS: $m / z=408.10[\mathrm{M}+\mathrm{H}]^{+} ;{ }^{1} \mathrm{H}-\mathrm{NMR}\left(300 \mathrm{MHz}, \mathrm{CDCl}_{3}\right): \delta 7.40-$ $7.38(\mathrm{~m}, 4 \mathrm{H}), 7.31-7.24(\mathrm{~m}, 4 \mathrm{H}), 7.20-7.15(\mathrm{~m}, 2 \mathrm{H}), 4.30(\mathrm{~s}$, $1 \mathrm{H}), 3.53-3.34(\mathrm{~m}, 5 \mathrm{H}), 3.31-3.25(\mathrm{~m}, 2 \mathrm{H}), 3.02-2.96(\mathrm{~m}$, $1 \mathrm{H}), 2.69(\mathrm{~m}, 2 \mathrm{H}), 2.02-1.93(\mathrm{~m}, 1 \mathrm{H}), 1.66-1.55(\mathrm{~m}, 2 \mathrm{H})$, 1.43-1.42 (m, 9H); ${ }^{13} \mathrm{C}-\mathrm{NMR}$ (75 MHz, $\left.\mathrm{CDCl}_{3}\right): \delta$ 154.6, 142.1, 128.6, 127.5, 127.2, 79.3, 78.6, 62.7, 62.4, 56.5, 55.7, 52.0, 51.7, 47.8, 44.4, 44.0, 32.4, 31.7, 28.6; HRMS (ESI): $[\mathrm{M}+\mathrm{H}]^{+}$calc'd for $\mathrm{C}_{25} \mathrm{H}_{33} \mathrm{~N}_{3} \mathrm{O}_{2} \mathrm{~m} / z$ 408.2651, found 408.2638 .

Tert-butyl 4-((1-benzhydrylazetidin-3-yl)amino)piperidine1-carboxylate (24). After stirring at $80{ }^{\circ} \mathrm{C}$ overnight, the mixture was placed in a $0-5{ }^{\circ} \mathrm{C}$ fridge for $16 \mathrm{~h}$, giving crystals. The filtrate was concentrated, and the residue was purified by silica gel column chromatography using $1: 9$ to 1:0 EtOAc / hexanes as eluent to give a solid $(122 \mathrm{mg}, 28 \%$ yield). LC-MS: $m / z=422.13[\mathrm{M}+\mathrm{H}]^{+} ;{ }^{1} \mathrm{H}-\mathrm{NMR}(300 \mathrm{MHz}$, $\left.\mathrm{CDCl}_{3}\right): \delta$ 7.40-7.37 (m, 4H), 7.29-7.24 (m, 4H), 7.20-7.15 (m, 2H), 4.30 (s, 1H), $3.98(\mathrm{~m}, 2 \mathrm{H}), 3.57-3.51(\mathrm{~m}, 3 \mathrm{H}), 2.75-$ $2.66(\mathrm{~m}, 4 \mathrm{H}), 2.62-2.52(\mathrm{~m}, 1 \mathrm{H}), 1.73-1.70(\mathrm{~m}, 2 \mathrm{H}), 1.43(\mathrm{~s}$, 9H), 1.28-1.14 (m, 2H); ${ }^{13} \mathrm{C}-\mathrm{NMR}$ (75 MHz, $\left.\mathrm{CDCl}_{3}\right): \delta 154.8$, $142.2,128.5,127.5,127.2,79.5,78.6,63.0,54.1,46.8,33.0$, 28.5; HRMS (ESI): $[\mathrm{M}+\mathrm{H}]^{+}$calc'd for $\mathrm{C}_{26} \mathrm{H}_{35} \mathrm{~N}_{3} \mathrm{O}_{2} \mathrm{~m} / \mathrm{z}$ 422.2808, found 422.2791 .

1-Benzhydryl-N-cyclobutylazetidin-3-amine (25). After stirring at $80{ }^{\circ} \mathrm{C}$ overnight, the mixture was placed in a $0-5{ }^{\circ} \mathrm{C}$ fridge for $16 \mathrm{~h}$, giving crystals. The filtrate was concentrated, and the residue was purified by silica gel column chromatography using 0:1 to $1: 9 \mathrm{MeOH} / \mathrm{DCM}$, then again us- ing 1:4 to $1: 1 \mathrm{EtOAc}+1 \% \mathrm{Et}_{3} \mathrm{~N} /$ hexanes $+1 \% \mathrm{Et}_{3} \mathrm{~N}$ as eluent to give an oil ( $80 \mathrm{mg}, 27 \%$ yield). LC-MS: $\mathrm{m} / z=293.00$ $[\mathrm{M}+\mathrm{H}]^{+} ;{ }^{1} \mathrm{H}-\mathrm{NMR}\left(300 \mathrm{MHz}, \mathrm{CDCl}_{3}\right): \delta$ 7.41-7.39 (m, 4H), 7.29-7.24 (m, 4H), 7.20-7.16 (m, 2H), $4.30(\mathrm{~s}, 1 \mathrm{H}), 3.52-$ $3.42(\mathrm{~m}, 3 \mathrm{H}), 3.23-3.16(\mathrm{~m}, 1 \mathrm{H}), 2.75-2.67(\mathrm{~m}, 2 \mathrm{H}), 2.16-$ $2.07(\mathrm{~m}, 2 \mathrm{H}), 1.74-1.54(\mathrm{~m}, 5 \mathrm{H}) ;{ }^{13} \mathrm{C}-\mathrm{NMR}\left(75 \mathrm{MHz}, \mathrm{CDCl}_{3}\right)$ : $\delta 142.3,128.5,127.5,127.2,78.7,62.5,52.9,47.1,31.7$, 15.1; HRMS (ESI): $[\mathrm{M}+\mathrm{H}]^{+}$calc'd for $\mathrm{C}_{20} \mathrm{H}_{24} \mathrm{~N}_{2} \mathrm{~m} / \mathrm{z}$ 293.2018, found 293.2018 .

1-Benzhydryl-N-(2-(5-methoxy-1H-indol-3-yl)ethyl)azetidin3-amine (26). After stirring at $80^{\circ} \mathrm{C}$ overnight, the mixture was placed in a $-20^{\circ} \mathrm{C}$ freezer for $16 \mathrm{~h}$, giving crystals. The filtrate was concentrated, and the residue was purified by silica gel column chromatography using 5:95 to 1:0 EtOAc $+1 \% \mathrm{Et}_{3} \mathrm{~N} /$ hexanes $+1 \% \mathrm{Et}_{3} \mathrm{~N}$ as eluent, then 1:99 to 5:95 $\mathrm{MeOH} / \mathrm{DCM}$ as eluent to give a solid (71 mg, 17\% yield). LC-MS: $m / z=412.10[\mathrm{M}+\mathrm{H}]^{+} ;{ }^{1} \mathrm{H}-\mathrm{NMR}\left(300 \mathrm{MHz}, \mathrm{CDCl}_{3}\right): \delta$ 7.93 (s, 1H), 7.39-7.36 (m, 4H), 7.32-7.23 (m, 5H), 7.19$7.15(\mathrm{~m}, 2 \mathrm{H}), 7.00(\mathrm{dd}, J=8.8,2.4 \mathrm{~Hz}, 2 \mathrm{H}), 6.86(\mathrm{dd}, J=8.8$, $2.3 \mathrm{~Hz}, 1 \mathrm{H}), 4.30(\mathrm{~s}, 1 \mathrm{H}), 3.83(\mathrm{~s}, 3 \mathrm{H}), 3.56-3.47(\mathrm{~m}, 3 \mathrm{H})$, 2.94-2.83 (m, 4H), 2.74-2.71 (m, 2H), $1.75(\mathrm{~s}, 1 \mathrm{H}) ;{ }^{13} \mathrm{C}-\mathrm{NMR}$ $\left(75 \mathrm{MHz}, \mathrm{CDCl}_{3}\right): \delta 154.0,142.2,131.6,128.5,127.8,127.5$, $127.2,122.9,113.5,112.4,112.0,100.7,78.5,61.7,56.0$, 48.8, 47.6, 26.1; HRMS (ESI): $[\mathrm{M}+\mathrm{H}]^{+}$calc'd for $\mathrm{C}_{27} \mathrm{H}_{29} \mathrm{~N}_{3} \mathrm{O}$ $\mathrm{m} / \mathrm{z} 412.2389$, found 412.2379 .

\section{ASSOCIATED CONTENT}

Supporting Information. Expanded experimental procedures and analytical data $\left({ }^{1} \mathrm{H},{ }^{13} \mathrm{C},{ }^{19} \mathrm{~F}\right.$ NMR, HPLC) for all new compounds (PDF). The Supporting Information is available free of charge on the Internet at http://pubs.acs.org.

\section{AUTHOR INFORMATION}

\section{Corresponding Author}

*matt@dunctonllc.com

\section{Author Contributions}

All authors have given approval to the final version of the manuscript.

Notes

The authors declare no competing conflicts.

\section{ACKNOWLEDGMENT}

We thank Vanessa Portman of Sygnature Discovery, BioCity, Pennyfoot Street, Nottingham, NG1 1GR, United Kingdom for high-resolution mass spectrometry.

\section{ABBREVIATIONS}

$\mathrm{ABB}$, azabicyclobutane; $\mathrm{Boc}_{2} \mathrm{O}$, di-tert-butyl dicarbonate; $\mathrm{DCM}$, dichloromethane; EtOAc, ethyl acetate; LDA, lithium diisopropylamide; $\mathrm{MeCN}$, acetonitrile; $\mathrm{MeOH}$, methanol; 5-MT, 5methoxytryptamine; TFMPP, 1-(3-trifluoromethylphenyl) piperazine; THF, tetrahydrofuran.

\section{REFERENCES}

(1) Clarke, E. T.; Johnson, J. R.; Robinson, R., Eds.; The Chemistry of Penicillin: Report on a Collaborative Investigation by American and British Chemists; Princeton University Press: Princeton, 1949, pp 1-1108.

(2) (a) Fridman, J. S.; Scherle, P. A.; Collins, R.; Burn, T. C.; Li, Y.; Li, J.; Covington, M. B.; Thomas, B.; Collier, P.; Favata, M. 
F.; Wen, X.; Shi, J.; McGee, R.; Haley, P. J.; Shepard, S.; Rodgers, J. D.; Yeleswaram, S.; Hollis, G.; Newton, R. C.; Metcalf, B.; Friedman, S. M.; Vaddi, K. Selective Inhibition of JAK1 and JAK2 Is Efficacious in Rodent Models of Arthritis: Preclinical Characterization of INCB028050. J. Immunol. 2010, 184, 5298-5307. (b) Rice, K. D.; Aay, N.; Anand, N. K.; Blazey, C. M.; Bowles, O. J.; Bussenius, J.; Costanzo, S.; Curtis, J. K.; Defina, S. C.; Dubenko, L.; Engst, S.; Joshi, A. A.; Kennedy, A. R.; Kim, A. I.; Koltun, E. S.; Lougheed, J. C.; Manalo, J. L.; Martini, J.; Nuss, J. M.; Peto, C. J.; Tsang, T. H.; Yu, P.; Johnston, S. Novel Carboxamide-Based Allosteric MEK Inhibitors: Discovery and Optimization Efforts toward XL518 (GDC-0973). ACS Med. Chem. Lett. 2012, 3, 416-421. (c) Huang, T.; Xue, C.; Wang, A.; Kong, L.; Ye, H.; Yao, W.; Rodgers, J. D.; Shepard, S.; Wang, H.; Shao, L.; Li, H.; Li, Q. Preparation of piperidin-4-yl azetidine derivatives as JAK1 inhibitors. W02011112662 (2011); Chem. Abstr. 155, 431880.

(3) (a) Sorbera, L. A.; Bayes, M.; Castaner, J.; Silvestre, J. Melagatran and Ximelagatran: Anticoagulant Thrombin Inhibitor. Drugs Fut. 2001, 26, 1155-1170. (b) Antonsson, T.; Gustafsson, D.; Hoffmann, K.; Nystrom, J.; Sorensen, H.; Sellen, M. Preparation of peptide derivatives as prodrugs of thrombin inhibitors. W09723499 (1997); Chem. Abstr. 127, 136080. (c) Kobayashi, T.; Inoue, T.; Nishino, S.; Fujihara, Y.; Oizumi, K.; Kimura, T. Novel 2-Amino-1,4dihydropyridine Calcium Antagonists. II. Synthesis and Antihypertensive Effects of 2-Amino-1,4-dihydropyridine Derivatives Having N,N-Dialkylaminoalkoxycarbonyl Groups at 3- and/or 5-Position. Chem. Pharm. Bull. 1995, 43, 797-817.

(4) (a) Han, Y.; Han, M.; Shin, D.; Song, C.; Hahn, H. Exploration of Novel 3-Substituted Azetidine Derivatives as Triple Reuptake Inhibitors. J. Med. Chem. 2012, 55, 8188-8192. (b) Yan, Q.; Wang, Y.; Zhang, W.; Li Y. Novel AzetidineContaining TZT-1027 Analogues as Antitumor Agents. Mar. Drugs 2016, 14: 85. Published 2016 Apr 28. doi: $10.3390 / \mathrm{md} 14050085$.

(5) (a) Takhi, M.; Sreenivas, K.; Reddy, C. K.; Munikumar, M.; Praveena, K.; Sudheer, P.; Rao, B. N. V. M.; Ramakanth, G.; Sivaranjani, J.; Mulik, S.; Reddy, Y. R.; Narasimha Rao, K.; Pallavi, R.; Lakshminarasimhan, A.; Panigrahi, S. K.; Antony, T.; Abdullah, I.; Lee, Y. K.; Ramachandra, M.; Yusof, R.; Rahman, N. A.; Subramanya, H. Discovery of Azetidine Based Ene-amides as Potent Bacterial Enoyl ACP Reductase (FabI) Inhibitors. Eur. J. Med. Chem. 2014, 84, 382-394. (b) Kirichok, A. A.; Shton, I. O.; Pishel, I. M.; Zozulya, S. A.; Borysko, P. O.; Kubyshkin, V.; Zaporozhets, 0. A.; Tolmachev, A. A.; Mykhailiuk, P. K. Synthesis of Multifunctional Spirocyclic Azetidines and Their Application in Drug Discovery. Chem. Eur. J. 2018, 24, 5444-5449.

(6) Zhang, X.; Hou, C.; Hufnagel, H.; Singer, M.; Opas, E.; McKenney, S.; Johnson, D.; Sui, Z. Discovery of a 4Azetidinyl-1-thiazoyl-cyclohexane CCR2 Antagonist as a Development Candidate. ACS Med. Chem. Lett. 2012, 3, 1039-1044.

(7) Chupak, L. S.; Flanagan, M. E.; Kaneko, T.; Magee, T. V.; Noe, M. C.; Reilly, U. Preparation of Erythromycin Macrolide Antibiotics and Their Use as Antibacterial and Antiprotozoal Agents. US20060135447 (2006); Chem. Abstr. 145, 83616.

(8) Han, M.; Song, C.; Jeong, N.; Hahn, H. Exploration of 3Aminoazetidines as Triple Reuptake Inhibitors by Bioisosteric Modification of 3- $\alpha$-Oxyazetidine. ACS Med. Chem. Lett. 2014, 5, 999-1004.

(9) George, D. M.; Breinlinger, E. C.; Friedman, M.; Zhang, Y.; Wang, J.; Argiriadi, M.; Bansal-Pakala, P.; Barth, M.; Duignan, D. B.; Honore, P.; Lang, Q.; Mittelstadt, S.; Potin, D.; Rundell, L.; Edmunds, J. J. Discovery of Selective and Orally
Bioavailable Protein Kinase C $\theta$ (PKC $\theta$ ) Inhibitors from a Fragment Hit. J. Med. Chem. 2015, 58, 222-236.

(10) For example: (a) Melloni, P.; Della Torre, A.; Meroni, M.; Ambrosini, A.; Rossi, A. C. Azetidine Derivatives of Tricyclic Antidepressant Agents. J. Med. Chem. 1979, 22, 183-191. (b) Ikee, Y.; Hashimoto, K.; Nakashima, M.; Hayashi, K.; Sano, S.; Shiro, M.; Nagao, Y. Synthesis and Antibacterial Activities of New Quinolone Derivatives Utilizing 1-Azabicyclo[1.1.0]butane. Bioorg. Med. Chem. Lett. 2007, 17, 942-945. (c) Ikee, Y.; Hashimoto, K.; Kamino, M.; Nakashima, M.; Hayashi, K.; Sano, S.; Shiro, M.; Nagao, Y. Synthesis of New Quinolone Antibiotics Utilizing Azetidine Derivatives Obtained from 1-Azabicyclo[1.1.0]butane. Chem. Pharm. Bull. 2008, 56, 346-356. (d) Boiteau, J.; Ouvry, G.; Arlabosse, J.; Astri, S.; Beillard, A.; BhurruthAlcor, Y.; Bonnary, L.; Bouix-Peter, C.; Bouquet, K.; Bourotte, M.; Cardinaud, I.; Comino, C.; Deprez, B.; Duvert, D.; Feret, A.; Hacini-Rachinel, F.; Harris, C. S.; Luzy, A.; Mathieu, A.; Millois, C.; Orsini, N.; Pascau, J.; Pinto, A.; Piwnica, D.; Polge, G.; Reitz, A.; Reverse, K.; Rodeville, N.; Rossio, P.; Spiesse, D.; Tabet, S.; Taquet, N.; Tomas, L.; Vial, E.; Hennequin, L. F. Discovery and Process Development of a Novel TACE Inhibitor for the Topical Treatment of Psoriasis. Bioorg. Med. Chem. 2018, 26, 945-956.

(11) (a) Gianatassio, R.; Lopchuk, J. M.; Wang, J.; Pan, C.; Malins, L. R.; Prieto, L.; Brandt, T. A.; Collins, M. R.; Gallego, G.M.; Sach, N. W.; Spangler, J. E.; Zhu, H.; Zhu, J.; Baran, P. S. Strain-release Amination. Science 2016, 351, 241-246. (b) Lopchuk, J. M.; Fjelbye, K.; Kawamata, Y.; Malins, L. R.; Pan, C.; Gianatassio, R.; Wang, J.; Prieto, L.; Bradow, J.; Brandt, T. A.; Collins, M. R.; Elleraas, J.; Ewanicki, J.; Farrell, W.; Fadeyi, O. O.; Gallego, G. M.; Mousseau, J. J.; Oliver, R.; Sach, N. W.; Smith, J. K.; Spangler, J. E.; Zhu, H.; Zhu, J.; Baran, P. S. Strain-Release Heteroatom Functionalization: Development, Scope, and Stereospecificity. J. Am. Chem. Soc. 2017, 139, 3209-3226.

(12) For examples of strain-release amination of ABB with substituted anilines and $\mathrm{Bn}_{2} \mathrm{NH}$ published prior to ref 11 see ref $10 \mathrm{~b}-\mathrm{c}$.

(13) Tatsuta, K.; Matsuba, A.; Inagaki, T. Method for Preparation of 3-Alkylaminoazetidines by Oxidation of 3Azetidinol Derivative to 3-Azetidinone Derivative and Reductive Amination with Alkylamine. JP2002255932 (2002); Chem. Abstr. 137, 216858.

(14) Representative examples: (a) Okutani, T.; Kaneko, T.; Masuda, K. Studies on Azetidine Derivatives. I. Synthesis of 3Substituted Azetidine Derivatives. Chem. Pharm. Bull. 1974, 22, 1490-1497. (b) Pryde, D. C.; Corless, M.; Fenwick, D. R.; Mason, H. J.; Stammen, B. C.; Stephenson, P. T.; Ellis, D.; Bachelor, D.; Gordon, D.; Barber, C. G.; Wood, A.; Middleton, D. S.; Blakemore, D. C.; Parsons, G. C.; Eastwood, R.; Platts, M. Y.; Statham, K.; Paradowski, K. A.; Burt, C.; Klute, W. The Design and Discovery of Novel Amide CCR5 Antagonists. Bioorg. Med. Chem. Lett. 2009, 19, 1084-1088.

(15) Representative examples: (a) Suzuki, Y.; Tsukamoto, K.; Hasegawa, Y.; Hiramatsu, Y. 3-Aminoazetidines or Their Salts. JP49109369 (1974); Chem. Abstr. 83, 9760. (b) Chen, Z.; Kolb, H. C.; Richardson, P.; Huang, Z. Preparation of 3Aminoazetidines as Building Blocks for Combinatorial Libraries. W09919297 (1999); Chem. Abstr. 130, 296695.

(16) For example, hydrogenation: Rodebaugh, R. M.; Cromwell, N. H. A Facile New Synthesis of dl-Azetidine-2-carboxylic Acid. J. Heterocyc. Chem. 1969, 6, 435-437.

(17) For example, 1-chloroethylchloroformate: Kharul, R. K.; Goswami, A.; Gite, A.; Godha, A. K.; Jain, M.; Patel, P. R. Convenient Synthesis of Structurally Novel 1,3Disubstituted Azetidine Derivatives. Synth. Commun. 2008, 38, 1703-1717. 
(18) Shepherd, R. G.; Adams, D. R.; Bodkin, C. D.; Cliffe, I. A.; Mansell, H. L.; Monck, N. J. Azetidinecarboxamide Derivatives for Treating CNS Disorders. W09937612 (1999); Chem. Abstr. 131, 116144.

(19) Duncton, M. A. J. unpublished results.

(20) (a) Duncton, M. A. J.; Estiarte, A. M.; Johnson, R. J.; Cox, M.; O'Mahony, D. J. R.; Edwards, W. T.; Kelly, M. G. Preparation of Heteroaryloxetanes and Heteroarylazetidines by Use of a Minisci Reaction. J. Org. Chem. 2009, 74, 6354-6357. (b) Duncton, M. A. J.; Estiarte, M. A.; Tan, D.; Kaub, C.; O'Mahony, D. J. R.; Johnson, R. J.; Cox, M.; Edwards, W. T.; Wan, M.; Kincaid, J.; Kelly, M. G. Preparation of Aryloxetanes and Arylazetidines by Use of an Alkyl-Aryl Suzuki Coupling. Org. Lett. 2008, 10, 3259-3262. (c) Pandey, A.; Samanta, S. K.; Duraiswamy, A. J.; Maciag, A. E.; Turner, D.; Duncton, M. A. J.; Kumari, V.; Renslo, A. R.; Low, E.; Brassard, C.; Adcock, H. V.; Hamza, D.; Onions, S. T. Synthesis of K-Ras Modulators Containing a Vinyl Sulfonamide Moiety. W02019204505 (2019); Chem. Abstr. 171, 518351. (d) Shishido, Y.; Nakao, K.; Nagayama, S.; Tanaka, H.; Duncton, M. A. J.; Cox, M.; Kincaid, J.; Sahasrabudhe, K.; EstiarteMartinez, M. A. Preparation of Bicyclic Amide Derivatives as Ion-channel Ligands. W02007133637 (2007); Chem. Abstr. 148, 11080.

(21) Anderson, A. G.; Lok, R. Synthesis of Azetidine-3carboxylic Acid. J. Org. Chem. 1972, 24, 3953-3955.

(22) It should be noted that references $10 \mathrm{~b}$ and $10 \mathrm{c}$ do describe aminations of ABB with primary anilines.

(23) Reference 11b, Supporting Information, pages S42-S43.

(24) Available from from ca. $\$ 200$ for $100 \mathrm{~g}$ (ca. $\$ 2$ per $1 \mathrm{~g}$ ).

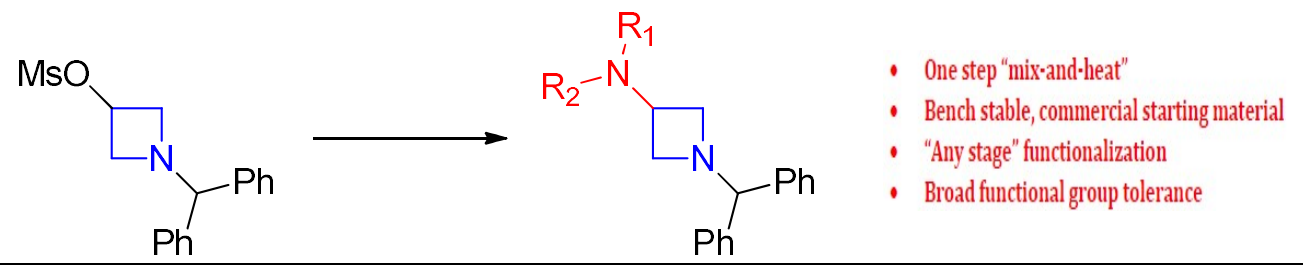

